Irish Math. Soc. Bulletin

Number 76, Winter 2015, 33-34

ISSN 0791-5578

\title{
COMPLETIONS OF PARTIAL MATRICES
}

\author{
JAMES MCTIGUE
}

This is an abstract of the $\mathrm{PhD}$ thesis Completions of Partial Matrices written by J. McTigue under the supervision of Rachel Quinlan at the School of Mathematics, Statistics, and Applied Mathematics, National University of Ireland, Galway and submitted in March 2015.

A partial matrix over a field $\mathbb{F}$ is a matrix whose entries are either elements of the field or independent indeterminates. A completion of a partial matrix is any matrix that results from assigning a field element to each indeterminate. The set of completions of an $m \times n$ partial matrix forms an affine subspace of $M_{m \times n}(\mathbb{F})$.

This thesis investigates partial matrices whose sets of completions satisfy particular rank properties - specifically partial matrices whose completions all have ranks that are bounded below and partial matrices whose completions all have the same rank. The maximum possible number of indeterminates in such partial matrices is determined, and the partial matrices that attain these bounds are fully characterized for all fields. These characterizations utilize a duality between properties of affine spaces of matrices that are related by the trace bilinear form.

Precise conditions (based on field order, rank and size) are provided to determine if a partial matrix whose completions all have rank $r$ must possess an $r \times r$ partial submatrix whose completions are all nonsingular.

Finally a characterization of maximal nonsingular partial matrices is provided - a maximal nonsingular partial matrix is a square partial matrix each of whose completions has full rank, with the property

2010 Mathematics Subject Classification. 15A83, 15A03.

Key words and phrases. Partial matrix, Completion, Rank.

Received on 13-12-2015.

Support from the College of Arts, Celtic Studies and Social Sciences at NUI Galway through a PhD scholarship is gratefully acknowledged. 
that replacement of any constant entry with an indeterminate results in a partial matrix having a singular completion.

School of Mathematics, Statistics and Applied Mathematics, NUi GALWAY

E-mail address, J. McTigue: mctiguejj@gmail.com 